\title{
System Level Study of LTE-Advanced Multiple Antenna System with Inter-Band Carrier Aggregation
}

\author{
Dana Salahalddin Abdalla \\ Communication Eng. Department \\ Technical college of Engineering \\ Sulaimani Polytechnic University \\ Sulaimani, Iraq \\ dana.abdala@spu.edu.iq
}

\author{
Salim Qadir Mohammed \\ Communication Eng. Department \\ Technical college of Engineering \\ Sulaimani Polytechnic University \\ Sulaimani, Iraq \\ Salim.muhammed@spu.edu.iq
}

\begin{abstract}
Spatial Multiplexing (SM) multiple antenna system and Carrier Aggregation (CA) are techniques introduced in Long Term Evolution- Advanced (LTE-Advanced) to support high data rates by increasing the number of transmission paths and the available bandwidth respectively. Therefore, in this study we evaluate the performance of LTE-Advanced physical downlink shared channel for single and SM multiple antenna systems in two different frequency bands. The radio channel is modelled using an enhanced Three-Dimensional (3D) international telecommunication union-radio communication sector channel model integrated with base station and user mobile 3D antenna patterns. Except the total received power, similar channel statistics are observed for both frequency bands. The study is performed considering $1 x 1,2 x 2,4 x 4$ antenna systems in a macro-cell urban environment at Component Carries (CC) of $2600 \mathrm{MHz}$ and $800 \mathrm{MHz}$ to model bands 7 and 20 of CA_7-20 respectively. The performance is evaluated in terms of throughput and SM gain for many base stations and user positions considering various modulation and coding schemes. We used the computationally efficient received bit information rate algorithm to compute the throughput as a function of channel structure and signal to noise ratio. As expected higher throughput is observed for the $800 \mathrm{MHz}$ band over the $2600 \mathrm{MHz}$ band. This is due to the higher total received power of the $800 \mathrm{MHz}$ band. The novel SM gain results show that the SM gain depends on the operating band and it's less than the number of spatial links. Moreover, the efficiency of inter-band $C A$ in increasing the data rates is a function cell radius and the number of spatial streams.
\end{abstract}

Keywords: Index Terms -LTE-Advanced, RBIR, Carrier Aggregation, MIMO.

\section{INTRODUCTION}

The evolution of Long Term Evolution (LTE) cellular communication systems to 4G LTE-Advanced is motivated by recent capacity demands. Bandwidth flexibility (from $1.4 \mathrm{MHz}$ to $20 \mathrm{MHz}$ ) is one of the main characteristics of LTE which allows radio access deployment in different frequency bands, each of which has their own unique characteristics. Even the $20 \mathrm{MHz}$
LTE bandwidth is not sufficient to support the target peak information rates of 1 Gbps in the Downlink (DL) channel and $500 \mathrm{Mbps}$ in the Uplink (UL) channel. Spatial Multiplexing (SM) Multi-In-Multi-Out (MIMO) antenna system and Carrier Aggregation (CA) are features that have been added to LTE-Advanced to achieve this goal. LTE-Advanced standard supports SM MIMO of up to 8 spatial streams in the DL and up to 4 spatial streams in the UL. In addition, LTE-Advanced allows the aggregation of two to five standard bandwidths using CA to produce a maximum bandwidth of $100 \mathrm{MHz}$ [1].

The 3GPP technical specification of LTE-Advanced supports aggregation of contiguous intra-bands and noncontiguous intra-bands and inter-bands. Each carrier frequency is called Component Carrier (CC). The propagation characteristics of each $\mathrm{CC}$ is different from the other $\mathrm{CCs}$ and this difference increases as the gap between the CCs increase. Table 1 shows a list of interband CA for the DL in the Release 11 of the LTEAdvanced 3GPP specification [2].

It can be noticed from the table that the gap between the $\mathrm{CCs}$ is greater than $1 \mathrm{GHz}$ and a maximum gap of $1845 \mathrm{MHz}$ can be observed for CA_7-20. This means a large difference between the total received power and the received level of the Signal to Noise Ratio (SNR). Having this difference in SNR between different CCs may lead to a difference in the receiver throughput which can degrade the efficiency of CA. This represents a big challenge for LTE-Advanced in achieving the target peak data rates.

Table 1: Inter-band CA in the DL (3GPP Rel 11)

\begin{tabular}{|c|c|c|c|c|}
\hline $\begin{array}{c}\text { E-UTRA } \\
\text { CA Band }\end{array}$ & $\begin{array}{c}\text { E-UTRA } \\
\text { Band }\end{array}$ & $\begin{array}{c}\text { Operating Band } \\
\text { MHz }\end{array}$ & $\begin{array}{c}\text { CC } \\
\text { MHz }\end{array}$ & $\begin{array}{c}\text { CCs Gap } \\
\text { MHz }\end{array}$ \\
\hline \multirow{2}{*}{ CA_1-5 } & 1 & $2110-2170$ & 2140 & \multirow{2}{*}{1260} \\
\cline { 2 - 4 } & 5 & $869-894$ & 880 & \\
\hline \multirow{2}{*}{ CA_4-13 } & 4 & $2110-2155$ & 2130 & \multirow{2}{*}{1380} \\
\cline { 2 - 4 } & 13 & $746-756$ & 750 & \\
\hline \multirow{2}{*}{ CA_7-20 } & 7 & $2620-2690$ & 2650 & \multirow{2}{*}{1845} \\
\cline { 2 - 4 } & 20 & $791-821$ & 805 & \\
\hline \multirow{2}{*}{ CA_2-17 } & 2 & $1930-1990$ & 1960 & \multirow{2}{*}{1220} \\
\cline { 2 - 4 } & 17 & $734-746$ & 740 & \\
\hline \multirow{2}{*}{ CA_3-20 } & 3 & $1805-1880$ & 1840 & \multirow{2}{*}{1035} \\
\cline { 2 - 4 } & 20 & $791-821$ & 805 & \\
\hline
\end{tabular}


The performance of inter-band CA between bands 5 and 7 are measured practically in [3] for $2 \times 2 \mathrm{SM}$ antenna system in a dense urban area with $187 \mathrm{~m}$ separation distance between the base stations. In [3], the BS transmit power of band 7 was set higher than the transmit power of band 5 to equalize the power spectrum density of the two bands. The study does not consider single antenna and 4x4 SM MIMO systems.

System-level simulation studies of communication systems is vital to understand and evaluate the overall performance of a system and optimize system parameters prior to standardization and real deployments. System level studies include many BSs and UEs, various modulation and coding schemes (MCS), and a number of scenarios. Performing system level studies using bit accurate physical layer simulators is time consuming. Instead the computational effective (RBIR) algorithm is a suitable alternative to the bit level simulator when studying the system level performance of OFDM based communication system [4]. Several abstraction models are available for OFDM based wireless communication systems. An RBIR abstraction model for WiMAX IEEE 802.16m WLAN system is developed in [5], and for LTEAdvanced downlink in [6]. Examples of abstract simulation models for MIMO-OFDM system without considering a specific communication standard includes the model of [7] which calculates the Minimum Mean Square Error (MMSE) iteratively at the receiver with Soft Interference Cancelation (SIC) and the model of [8] for maximum likelihood detector.

We study in this paper the performance of LTE-Advanced SM MIMO antenna system in the DL. We evaluate the performance at carrier frequencies of $2600 \mathrm{MHz}$ and $800 \mathrm{MHz}$ to model bands 7 and 20 of CA_7-20 respectively. The RBIR abstraction technique proposed in [6] used in this study to quantify the Packet Error Rate (PER) and throughput in the DL for LTE-Advanced $1 \mathrm{x} 1$, $2 \times 2$, and $4 \times 4$ antenna configurations. Obtained simulation results are for large numbers of urban users in an interference-free scenario. The enhanced 3D ITU-R channel model, integrated with $3 \mathrm{D}$ radiation patterns for the macro-cell base stations (BS) and the user equipment (UE) antennas obtained from measurement, is used to model the multipath fading wireless channel between the BSs and the UEs. Each BS-UE link is characterized by pathloss, shadowing, and multipath effect in the enhanced 3D ITU-R model.

The remainder of the paper is as follows: The System model is defined in Section 2. Section 3 describes the RBIR technique and presents the validation results for single and multiple antenna systems. Simulation results for system level performance are shown in Section 4 and Section 5 draw conclusions.

\section{System Model}

\section{A. Enhanced 3D ITU-R Channel Modeling Process}

The communication channels in this paper are generated using the enhanced 3D International Telecommunication Union-Radio communication sector (ITU-R) channel model [9]. The ITU-R model is a Geometry based Stochastic Channel Model (GSCM) that needs various randomness levels to model many BS-UE channels. As shown in figure 1 , the channel generation process of the enhanced 3D ITU-R GSCM [10] can be described using six steps. These are classified into three phases: 1) UE parameters, 2) generation of Large Scale Parameters (LSPs) and 3) generation of channel impulse response.

The user parameter part (step1) is used to setup simulation parameters, such as the type of environment, the numbers of BSs and UEs, the directions and speeds of the UE, and the propagation condition Line Of Sight (LOS) and NonLOS (NLOS). In this phase, the users also can supply the antenna patterns at both the BS and UE, the spacing and orientations of the antenna elements. The second part of the channel creation process is the propagation parameter generation, which consists of Path Loss (PL), Shadow Fading (SF) calculation and the generation of LSPs and Small Scale Parameters (SSPs) for the channel. LSPs are generated based on a pre-defined Probability Distribution Functions (PDF) with specific mean and standard deviation.

These include the Root Mean Square (RMS) Delay Spread (DS), the RMS Angel of Arrival (AoA) and Angle of departure (AoD) in both azimuth and elevation, the KFactor and the SF. The de-correlation distances and cross correlations are calculated for the generated LSPs. For more details on these parameters please refer to [11]. The SSPs are now generated based on the LSPs from step 3 . The SSPs represent the information associated with each Multipath Component (MPC). This include the phase, delay, angular information for each individual cluster and ray within the cluster. This is performed based on the predefined PDFs.

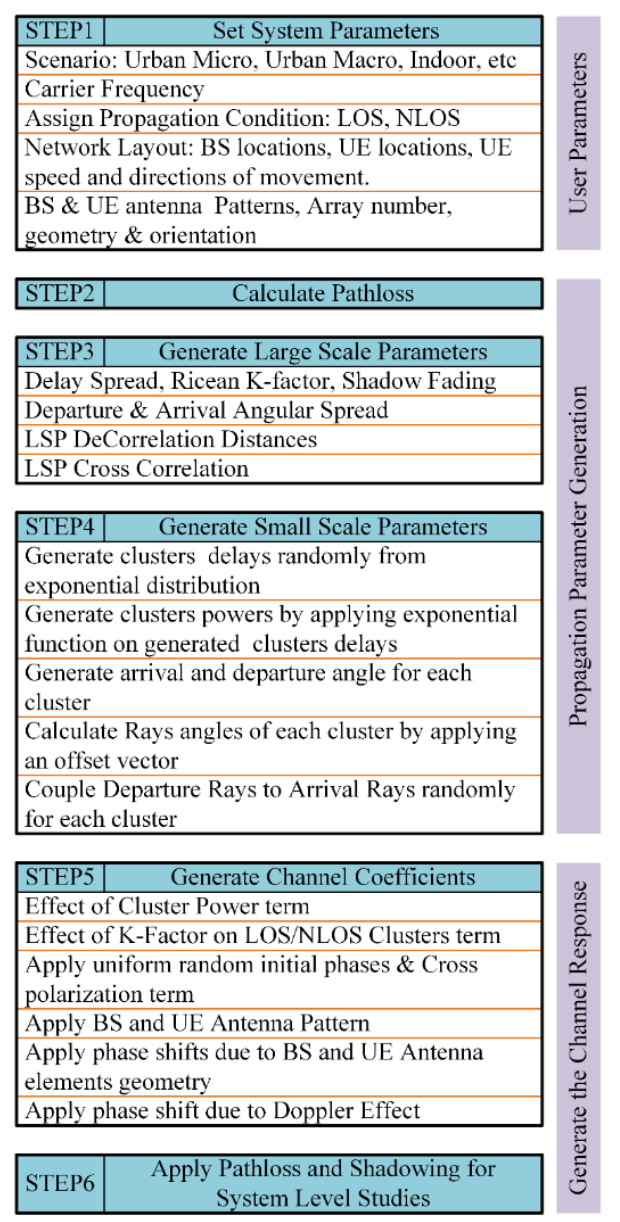

Figure 1: Enhanced 3D ITU-R channel modelling 
Step 5 represents the generation of the channel impulse response in time domain. This includes generating random phases for the rays within cluster and apply the cross-polarization effect between antenna elements. Then the Doppler effect is added in case of mobility. Finally, in step 6, PL and SF values are applied to the channel impulse responses. This stage enables system level studies to be performed. The power radiation pattern of the antenna used in this paper are shown in figure 2 for the macro BS and UE. These are obtained from anechoic chamber measurements performed at the University of Bristol [12]. All patterns are captured in 3D and consists information about the phase, polarization and directivity. Table 2 summarizes the antenna patterns parameters. The code file of the channel model is available in [13] and the channel model parameters are summarized in table 2.

Table 2: System Parameters

\begin{tabular}{|c|c|c|}
\hline \multicolumn{2}{|l|}{ Parameter } & Value \\
\hline \multicolumn{2}{|l|}{ LTE Bandwidth } & $10 \mathrm{MHz}$ \\
\hline \multicolumn{2}{|c|}{ No. of Subcarrier ( $\left.\mathrm{N}_{\mathrm{subc}}\right)$} & 600 \\
\hline \multicolumn{2}{|c|}{ No. of OFDM Symbols } & 7 \\
\hline \multicolumn{2}{|l|}{$\mathrm{T}_{\text {slot }}(\mathrm{ms})$} & 0.5 \\
\hline \multicolumn{2}{|l|}{ Carrier Frequency } & $800 \mathrm{MHz}, 2.6 \mathrm{GHz}$ \\
\hline \multicolumn{2}{|l|}{ Environments } & Urban (ITU-R scenario 4) \\
\hline \multicolumn{2}{|l|}{ Cellular Deployment } & 3-sector hexagonal grid \\
\hline \multicolumn{2}{|l|}{ Number of BS } & 20 \\
\hline \multicolumn{2}{|l|}{ Number of Sectors } & 3 \\
\hline \multicolumn{2}{|c|}{ Number of UEs per Sector } & 300 \\
\hline \multicolumn{2}{|l|}{ BS heights (m) } & Range of 7-77 $\mathrm{m}$ above ground \\
\hline \multicolumn{2}{|c|}{ Total Number of UE's } & 18000 \\
\hline \multicolumn{2}{|l|}{ UE height $(\mathrm{m})$} & 1.5 \\
\hline \multicolumn{2}{|l|}{ UE locations } & $50-1000 \mathrm{~m}$ from BS \\
\hline \multicolumn{2}{|c|}{ BS transmit power $(\mathrm{dBm})$} & 43 \\
\hline \multicolumn{2}{|l|}{ BS antenna downtilt } & $10^{\circ}$ \\
\hline \multicolumn{2}{|l|}{ MIMO scheme } & $2 \times 2,4 \times 4$ Spatial Multiplexing \\
\hline \multirow{2}{*}{ Antenna Type } & $\mathrm{BS}$ & $\begin{array}{l}\text { Uniform linear array with } 6 \text { dual } \\
\text { polarized patches }\end{array}$ \\
\hline & UE & $\begin{array}{l}\text { NOKIA mobile phone antenna } \\
\text { (omni-directional) }\end{array}$ \\
\hline \multirow{2}{*}{$\begin{array}{l}\text { 3dB Beamwidth } \\
\text { Azimuth/Elevation }\end{array}$} & $\mathrm{BS}$ & $65^{\circ} / 15^{\circ}$ \\
\hline & UE & $360^{\circ} / 36^{\circ}$ \\
\hline \multirow{2}{*}{$\begin{array}{l}\text { Antenna element } \\
\text { spacing }\end{array}$} & $\mathrm{BS}$ & $10 \lambda$ \\
\hline & UE & $0.5 \lambda$ \\
\hline
\end{tabular}

\section{B. Channel Model Propagation Staistics}

The propagation statistics of the enhanced 3D ITU-R channel model for urban environment are shown in figure 3, which includes the Cumulative Distribution Function (CDF) of the path loss, the K-factor, the RMS values of the DS, azimuth departure angle (AoDaz) spread, elevation departure angle (AoDel) spread, azimuth arrival angle (AoAaz) spread, and elevation arrival angle (AoAel) spread. Since the study is performed for the downlink, the angles of departure refer to the base station side and the angle of arrival represents the user mobile side.
Comparing the results of figure 3 in terms of component carrier frequency, considerably higher total received power occurs at $800 \mathrm{MHz}$. Both frequencies have approximately the same delay and angular spreads as well as $\mathrm{K}$-factor.

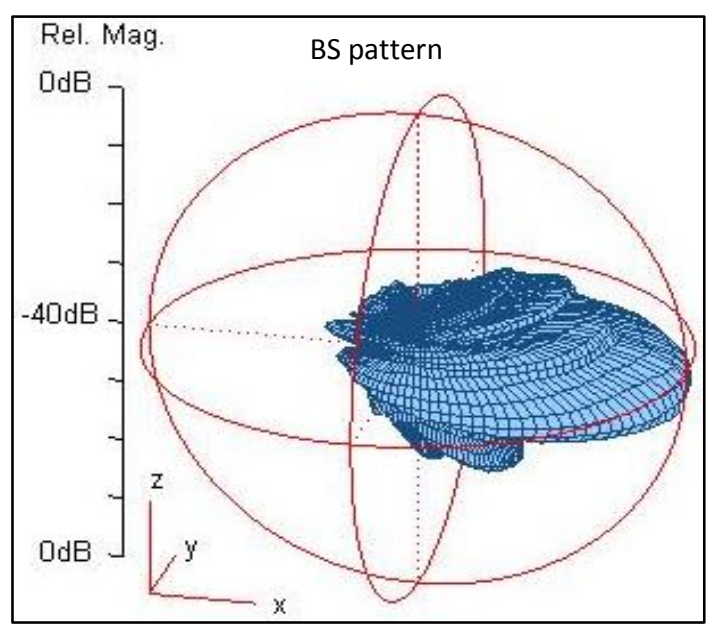

(a) Base station (BS) pattern

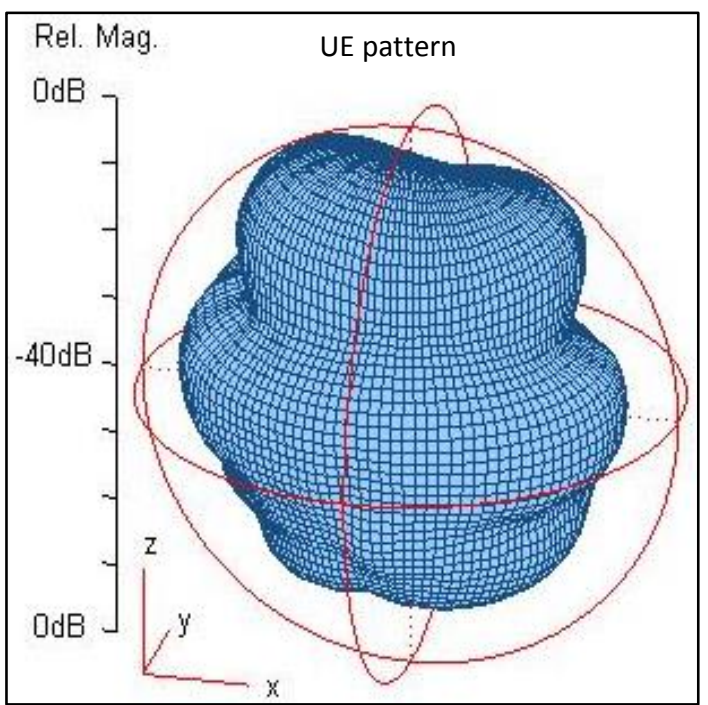

(b) User equipment (UE) pattern

Figure 2: BS and UE obtained radiation patterns [12]

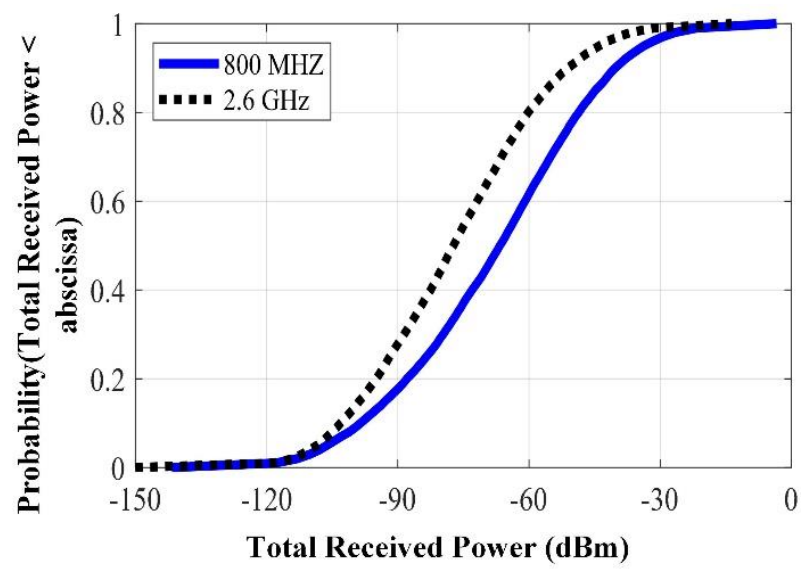

a) Average received power 


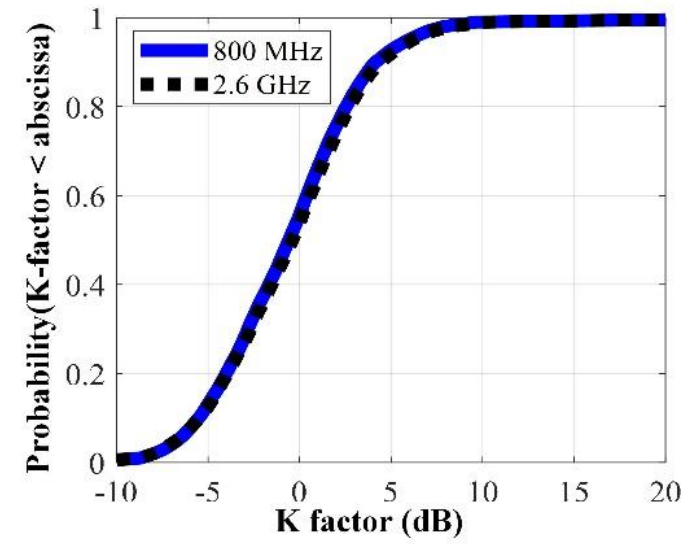

b) K-factor

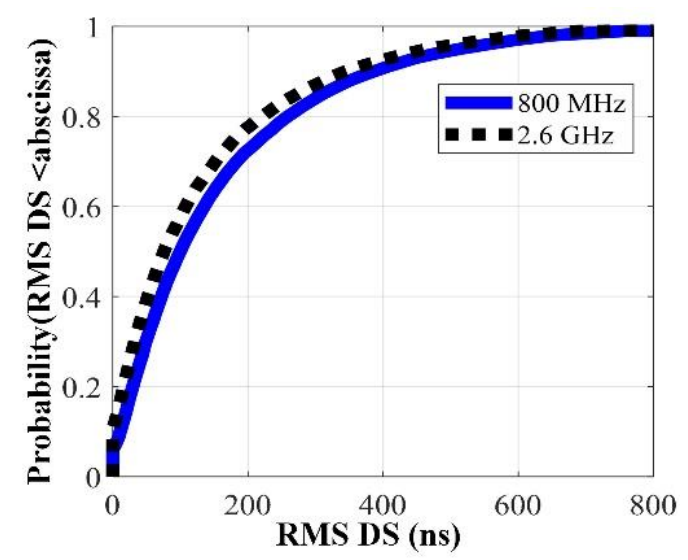

c) Delay spread

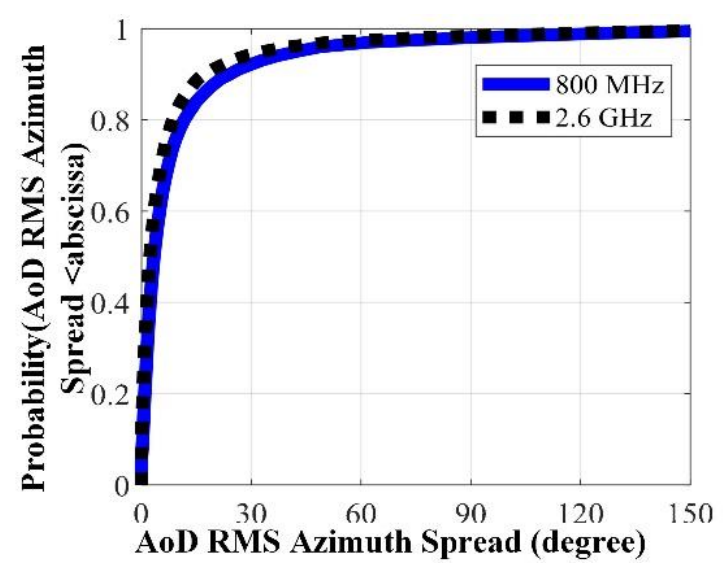

d) AoDaz

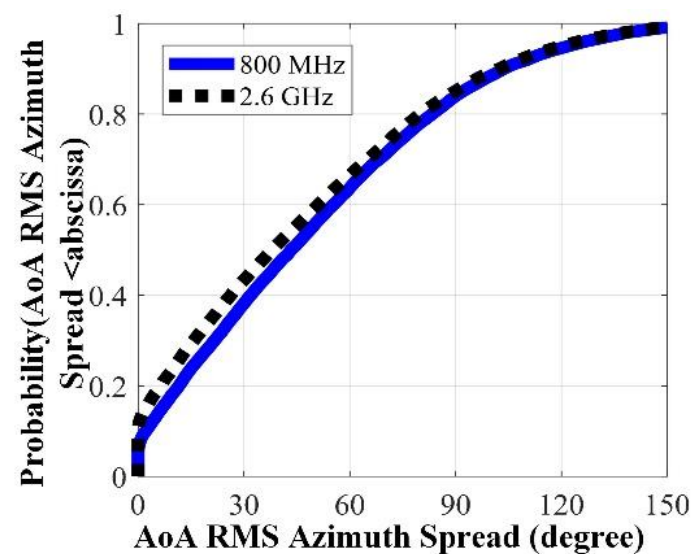

e) AoAaz

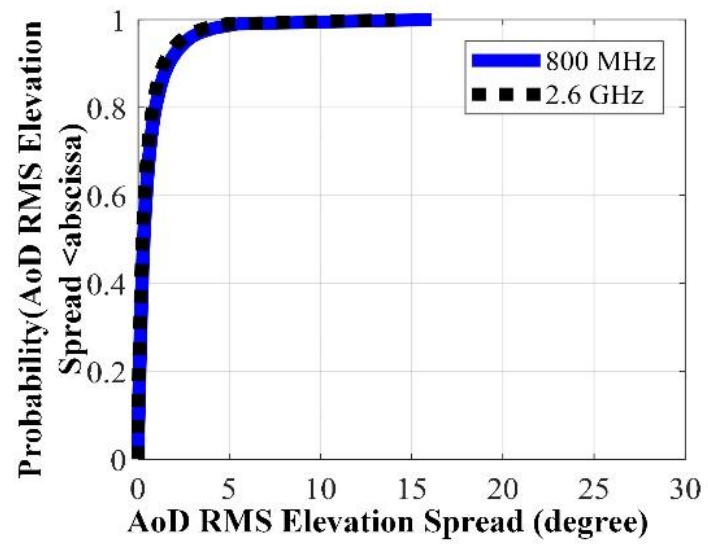

f) AoDel

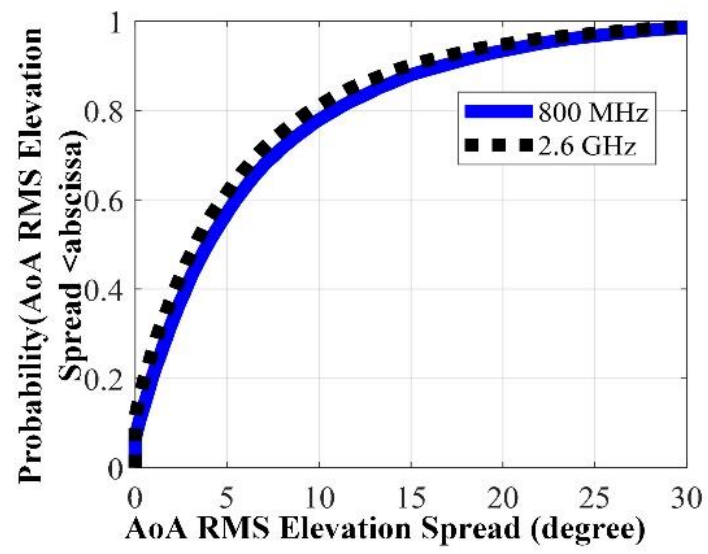

g) AoAel

Figure 3: Propagation statistics of the enhanced 3D ITU-R channel model

\section{Network Layout and SNR Calculation}

The LTE-Advanced system is based on a hexagonal macro cellular deployment shown in figure 4. Every BS site covers three sectors in urban environment (ITU scenario 4) with a cell radius of $1 \mathrm{~km}$ for $20 \mathrm{BSs}$ assuming different BS antenna heights. Within each sector 300 UEs were randomly scattered at street level. The system was modelled at CCs of $800 \mathrm{MHz}$ and $2600 \mathrm{MHz}$. The system parameters of this paper are listed in table 2 . The average SNR at each UE location is calculated using (1):

$$
S N R_{U E}=\frac{P_{U E}}{P_{A W G N}}
$$

In (1), $P_{U E}$ represents the total received signal power at UE location and $P_{A W G N}$ is determined using (2) which represents the Additive White Gaussian Noise(AWGN):

$$
P_{A W G N}=K * T * B_{\text {effec }} * F_{\text {linear }}
$$

In (2) $\mathrm{K}$ represents Boltzmann's constant, $\mathrm{T}$ is the temperature in Kelvin, $\mathrm{B}_{\text {effec }}$ which is equal to $9 \mathrm{MHz}$ and represents the number of subcarriers multiplied by the subcarrier frequency. In this case 600 subcarriers multiplied by $15 \mathrm{kHz}$. $\mathrm{F}_{\text {linear }}$ is the noise figure (linear value). In this paper a temperature value of 288 Kelvin and a noise figure value of $\mathrm{F}_{\mathrm{dB}}=9 \mathrm{~dB}$ is chosen based on [14]. 


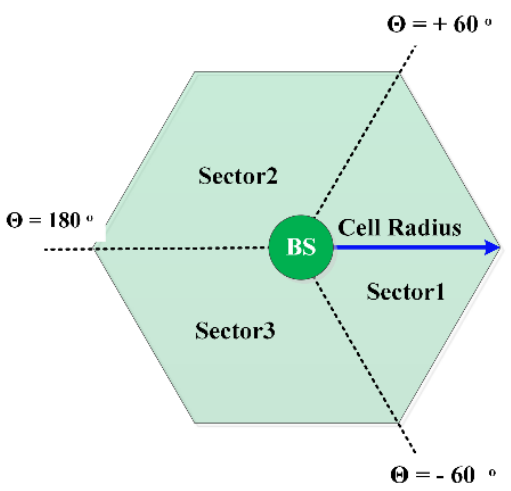

Figure 4: The macro cell topology

\section{Throughput Estimation}

The total numbers of UEs included in this study are 18000 (20 BSs, 900 UEs each). For every BS-UE channel, the impulse response is generated using the enhanced $3 \mathrm{D}$ ITU-R GSCM, converted into the frequency domain, and used as input into our physical downlink shared channel (PDSCH) RBIR abstraction engine to estimate the rate of packet error for $10 \mathrm{MCSs}$ at an SNR value calculated using (1). Note that the same process is followed when estimating the performance using bit accurate physical layer simulations for the validation study of the RBIR abstract engine in section 3 .

A link adaptation algorithm is applied that choses the MCS that provide the highest Throughput (THR) of every link. The LTE-Advanced PDSCH throughput is calculated using (3) assuming perfect channel knowledge at the receiver [15].

$$
T H R_{M C S}=R_{M C S} *\left(1-P E R_{M C S}\right)
$$

In (3) $\mathrm{R}_{\text {MCS }}$ can be calculated in (Mbps) using (4) as a function of modulation order $\left(\mathrm{k}_{\mathrm{m}}\right)$, the coding rate $\left(\mathrm{R}_{\mathrm{C}}\right)$, the active subcarriers $\left(\mathrm{N}_{\text {subc }}\right)$ and the OFDM symbols $\left(\mathrm{N}_{\text {symb }}\right)$ in the time slot $\left(\mathrm{T}_{\text {slot }}\right)$. Table 2 summarizes the system parameters used here while table 3 lists the value of $\mathrm{R}_{\mathrm{MCS}}$ for each considered MCS mode.

$$
R_{M C S}=\frac{k_{m} \cdot R_{c} \cdot N_{s u b c} \cdot N_{s y m b}}{T_{\text {slot }}}
$$

\section{RBIR ALgORITHM AND VALIDATION RESULTS}

As mentioned earlier RBIR technique is a computational efficient technique used instead to bit level simulation when studying the system level performance of OFDM based communication. Therefore, this section first describes the RBIR algorithm (in sub-section A), then presents BER versus SNR performance validation result of [6] for single and multiple antenna systems in sub-section $\mathrm{B}$ and sub-section $\mathrm{C}$ respectively.

\section{A. RBIR Algorithm}

The following describes the steps required in RBIR technique [5]:

Step1: For a specific MCS, SNR, and channel sample, calculate an SNR vector which represents SNR variations across the OFDM sub-carriers as results of frequency selective fading.
Step2: The SNR vector is converted to a single effective value (ESNR) using the effective mapping (ESM) of (5).

$$
\operatorname{ESNR}=\Phi_{m}^{-1}\left\{\frac{1}{N_{\text {subc }} \cdot N_{\text {stream }}} \sum_{n=1}^{N_{\text {subc }}} \sum_{k=1}^{N_{\text {stream }}} \Phi\left(S N R_{n, k}\right)\right\}
$$

Where, $\mathrm{SNR}_{\mathrm{n}, \mathrm{k}}$ is the SNR of the nth sub-carrier for the kth spatial link. $\mathrm{m}$ is the modulation type, $\mathrm{N}_{\text {stream }}$ is the number of spatial streams, and $\Phi($.$) is an invertible function. For$ Mutual Information (MI) ESM approach, $\Phi($.$) is defined$ as the Symbol Information (SI) as given in (6).

$$
S I(\gamma, m)=E_{X Y}\left\{\log _{2} \frac{P(Y \mid X, \gamma)}{\sum_{X} P(X) P(Y \mid X, \gamma)}\right\}
$$

In (6) $Y$ is the symbol received for an SNR value of $\gamma$ and $P(Y \mid X, \gamma)$ is the AWGN probability density for noise-free transmit symbol $X . P(X)$ is selected as $1 / m$.

Step3: The instantaneous (BER) or (PER) can be computed by mapping the ESNR using a look-up-table containing BER or PER performance versus SNR for an AWGN channel. This table can be obtained from bit accurate simulation.

Step4: Calculate the final BER or PER performance for a specific SNR and MCS by averaging the instantaneous BER or PER across all channel samples. figure 5 shows the block diagram of the transmitter for LTE-Advanced

\begin{tabular}{|c|c|c|c|}
\hline MCS & Modulation & Code rate & $\mathbf{R}_{\text {MCS }}$ (Mbps) for SISO \\
\hline 1 & \multirow{4}{*}{$\begin{array}{c}\text { QPSK } \\
{\left[\mathrm{k}_{\mathrm{m}}=2\right]}\end{array}$} & $1 / 3$ & 5.6 \\
\hline 2 & & $1 / 2$ & 8.4 \\
\hline 3 & & $2 / 3$ & 11.2 \\
\hline 4 & & $4 / 5$ & 14.44 \\
\hline 5 & \multirow{3}{*}{$\begin{array}{c}16 \text { QAM } \\
{\left[\mathrm{k}_{\mathrm{m}}=4\right]}\end{array}$} & $1 / 2$ & 16.8 \\
\hline 6 & & $2 / 3$ & 22.4 \\
\hline 7 & & $4 / 5$ & 26.88 \\
\hline 8 & \multirow{3}{*}{$\begin{array}{c}64 \text { QAM } \\
{\left[\mathrm{k}_{\mathrm{m}}=6\right]}\end{array}$} & $2 / 3$ & 33.6 \\
\hline 9 & & $3 / 4$ & 37.8 \\
\hline 10 & & $4 / 5$ & 40.32 \\
\hline
\end{tabular}
PDSCH bit accurate simulator. The reverse operations are performed at the receiver.

Table 3: List of MCS modes and peak error free bit rates

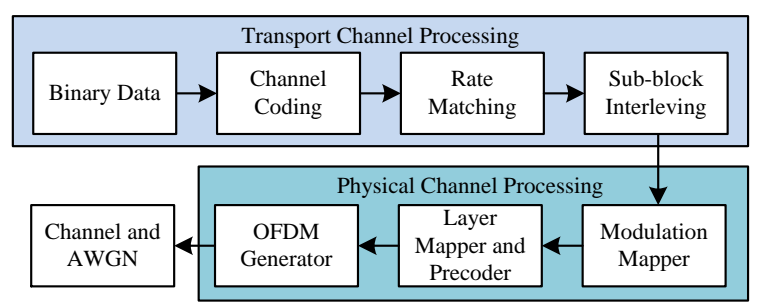

Figure 5: Block diagram of the LTE-Advanced downlink transmitter.

\section{B. Single Antenna System:}

This section presents BER versus SNR performance obtained in [6] using the RBIR and bit accurate simulators to prove the precision of the RBIR concept for single antenna system. The simulation results are for two UE locations; location1 with LOS (blue colour graphs) and location2 with NLOS (brown graphs). The channel 
parameters at these two locations are listed in table 4. As mentioned previously, 10 MCS modes considered in this paper. However, for clarity figure 6 shows the simulation results for just 3 MCS modes. A good match can be observed between the bit level and RBIR abstraction simulations.

\section{Spatial Multiplexing Multiple Antenna System}

This section shows BER versus SNR performance validation results of the RBIR abstraction engine for SM MIMO antenna system. The verification was performed in [6] for four different cases, 2x2 and 4x4 SM MIMO each with low and medium spatial correlation. The low and medium spatial correlations are defined by the 3GPP technical specification [16] as a combination of spatial correlation factors $(\alpha)$ and $(\beta)$ for BS and UE respectively as summarised in table 5 . Figure 7 shows the simulation results for just 2 MCS modes (for clarity). The channel parameters at the UE locations for the four cases are listed in table 6. A good match can be observed between the bit level and RBIR abstraction simulations.

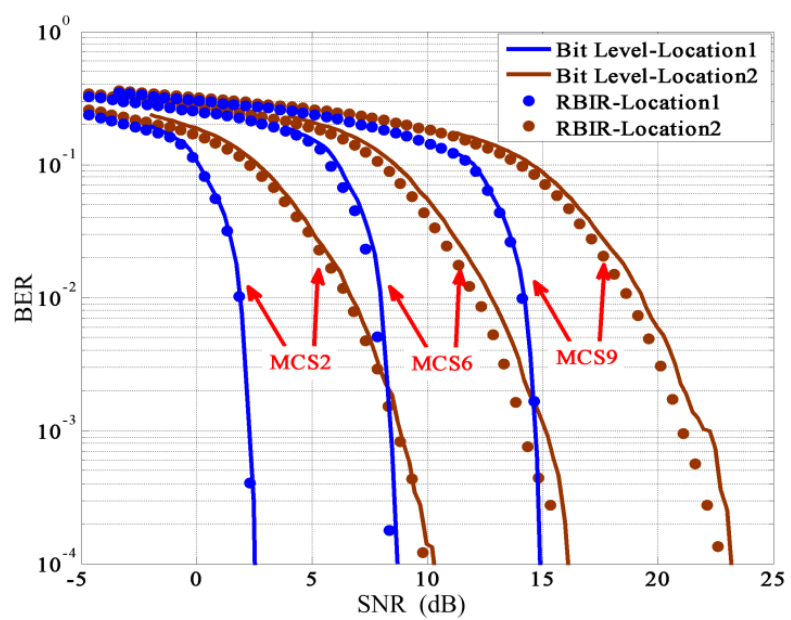

Figure 6: BER comparison between Bit level and RBIR simulator for single antenna system [6]

Table 4: Channel model parameters for the two UE locations in figure 6

\begin{tabular}{|l|c|c|}
\hline \multicolumn{1}{|c|}{ Parameter } & Location1 & Location2 \\
\hline Channel length (samples) & 7 & 34 \\
\hline K-factor (dB) & 17.2 & -9 \\
\hline RMS Delay Spread(ns) & 21.2 & 765.7 \\
\hline AoD RMS Azimuth & 0.73 & 3.17 \\
\hline AoD RMS Elevation & 0.001 & 0.53 \\
\hline AoD RMS Azimuth & 8.14 & 70 \\
\hline AoD RMS Elevation & 3.13 & 17.27 \\
\hline
\end{tabular}

Table 5: 3GPP BS and UE spatial correlation factors

\begin{tabular}{|l|c|c|c|}
\hline \multirow{2}{*}{ Parameter } & \multicolumn{3}{|c|}{ Value } \\
\cline { 2 - 4 } & $\begin{array}{c}\text { Low } \\
\text { Correlation }\end{array}$ & $\begin{array}{c}\text { Medium } \\
\text { Correlation }\end{array}$ & $\begin{array}{c}\text { High } \\
\text { Correlation }\end{array}$ \\
\hline $\begin{array}{l}\text { BS Spatial } \\
\text { Correlation }(\alpha)\end{array}$ & 0 & 0.3 & 0.9 \\
\hline $\begin{array}{l}\text { UE Spatial } \\
\text { Correlation }(\beta)\end{array}$ & 0 & 0.9 & 0.9 \\
\hline
\end{tabular}

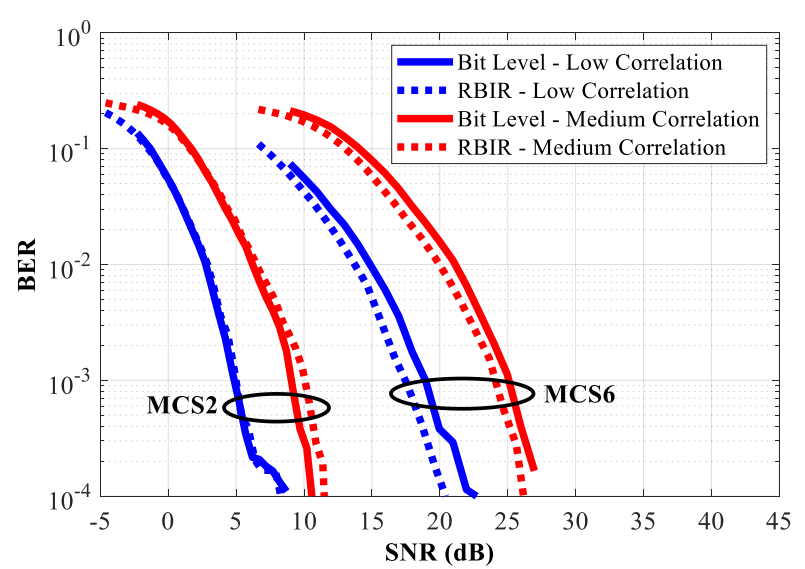

a) $2 \times 2 \mathrm{SM}$ MIMO

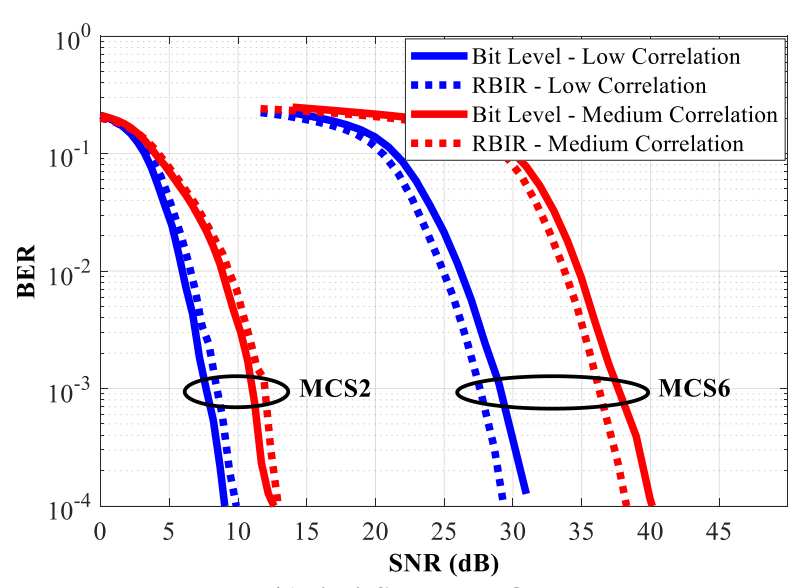

b) $4 \times 4$ SM MIMO

Figure 7: BER comparison results of Bit level and RBIR simulator of $2 \times 2$ and $4 \times 4$ SM MIMO for MCS2 and MCS6 [6]

Table 6: Channel model parameters for the four cases of figure 7

\begin{tabular}{|l|c|c|c|c|}
\hline \multicolumn{1}{|c|}{ Parameter } & Case1 & Case2 & Case3 & Case4 \\
\hline MIMO & $2 \times 2$ & $2 \times 2$ & $4 \times 4$ & $4 \times 4$ \\
\hline Correlation value & low & med & low & med \\
\hline$\alpha$ & 0.002 & 0.29 & 0.06 & 0.28 \\
\hline$\beta$ & 0.026 & 0.89 & 0.02 & 0.89 \\
\hline K-factor (dB) & -8.8 & -1.4 & -6.5 & 0.7 \\
\hline RMS Delay Spread(ns) & 38.9 & 146.6 & 198.8 & 61.0 \\
\hline AoD RMS Azimuth & $6.5^{\circ}$ & $3.2^{\circ}$ & $9.0^{\circ}$ & $4.9^{\circ}$ \\
\hline AoD RMS Elevation & $1.0^{\mathbf{o}}$ & $0.4^{\circ}$ & $1.6^{\circ}$ & $0.6^{\circ}$ \\
\hline AoD RMS Azimuth & $132.5^{\mathbf{o}}$ & $23.9^{\circ}$ & $30.1^{\circ}$ & $63^{\circ}$ \\
\hline AoD RMS Elevation & $17.4^{\circ}$ & $0.5^{\circ}$ & $2.4^{\circ}$ & $14^{\circ}$ \\
\hline
\end{tabular}

\section{Simulation ReSUltS}

This section presents the simulation results of LTE-Advanced PDSCH. The simulation is performed for $1 \times 1,2 \times 2,4 \times 4$ antenna systems in macro-cell urban environment at CCs of $800 \mathrm{MHz}$ and $2600 \mathrm{MHz}$ each with $10 \mathrm{MHz}$ bandwidth. figure 8 shows the CDF of throughput in (Mbps) for all UEs in the 20 macro-cells considered. It is obvious from figure 8 that the throughput at $800 \mathrm{MHz}$ is higher than that at $2600 \mathrm{MHz}$. This is due to the relative higher total transmit power for the $800 \mathrm{MHz}$ band compared to the $2600 \mathrm{MHz}$ band (see figure 3a). For instance, at $800 \mathrm{MHz}, 30 \%$ of UEs provide a throughput less than or equal to $140 \mathrm{Mbps}$. However, at $2600 \mathrm{MHz}$ 
the number of UEs that provide less than $140 \mathrm{Mbps}$ throughput increase to $60 \%$. This means that the $800 \mathrm{MHz}$ gains $30 \%$ higher throughput compared to the $2600 \mathrm{MHz}$ band.

In figure 8, the throughput increases with increasing the number of antennas. However, this increase has no linear relationship with the increase in number of spatial streams (two and four in the $2 \times 2$ and $4 \times 4$ MIMO system respectively). As shown in figure 9, the reason for this non-linearity is that not all the UEs have a SM gain equal to the number of MIMO streams. For example, considering the $800 \mathrm{MHz}$ CDF graphs of figure 9, around $58 \%$ of the UEs experience a SM gain less than two for 2x2 MIMO system. For 4x4 MIMO system, around 68\% of the UEs have SM gain less than 4.

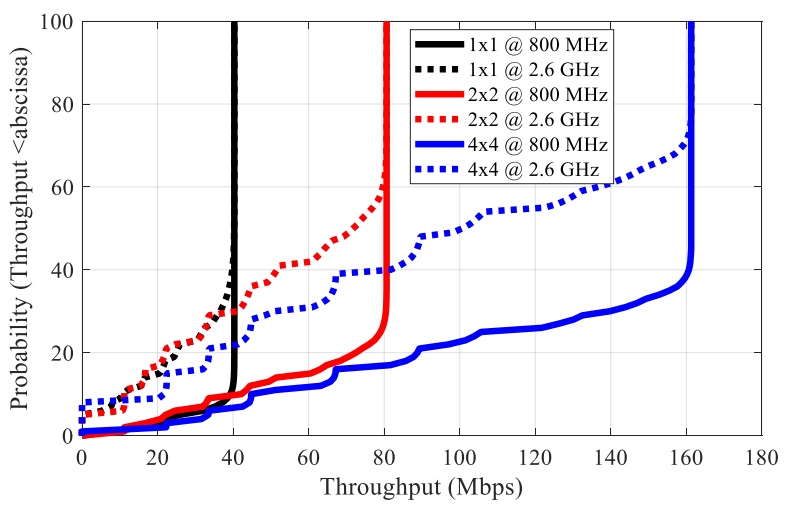

Figure 8: CDF of UEs throughput for all UEs and BSs.

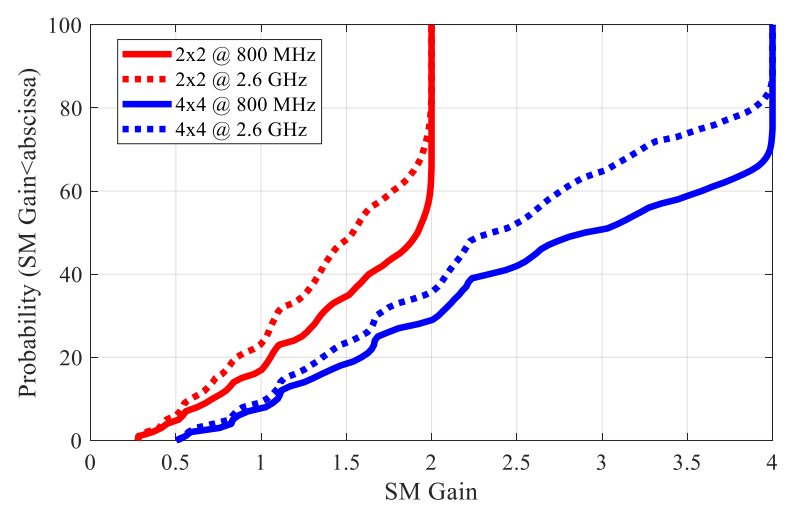

Figure 9: CDF of downlink SM gain for all UEs

Finally, figure 10 shows the throughput scattered plot for one cell for the three-different antenna configuration at both frequencies. The figure illustrates the dependency of the carrier aggregation efficiency on the number of antenna elements. The coverage area of the $4 \times 4$ MIMO system significantly decreased compared to the $2 \times 2$ and $1 \mathrm{x} 1$ antenna system. This is due to increase interstream interference of $4 \times 4$ antenna element compared to the $2 \times 2$ and $1 \times 1$. The increase in the throughput of $4 \times 4$ and $2 \times 2$ MIMO systems comes on the cost of reduced coverage area compared to the single antenna system. figure 10 also shows that efficient CA can be achieved by choosing approximately cell radiuses of $800 \mathrm{~m}, 500 \mathrm{~m}, 250 \mathrm{~m}$ for $1 \times 1,2 \times 2$, and $4 \times 4$ antenna systems respectively.

To maintain good CA efficiency, the cell radius is decreased $20 \%$ in SISO, $50 \%$ in 2x2 MIMO, $75 \%$ in $4 \times 4$ MIMO. In other words, the efficiency of CA decreases as the number of antenna elements increases. As mentioned previously, this is due to the worst PER performance of multiple antenna systems because the inter stream interference increases as the number of antenna elements increases.
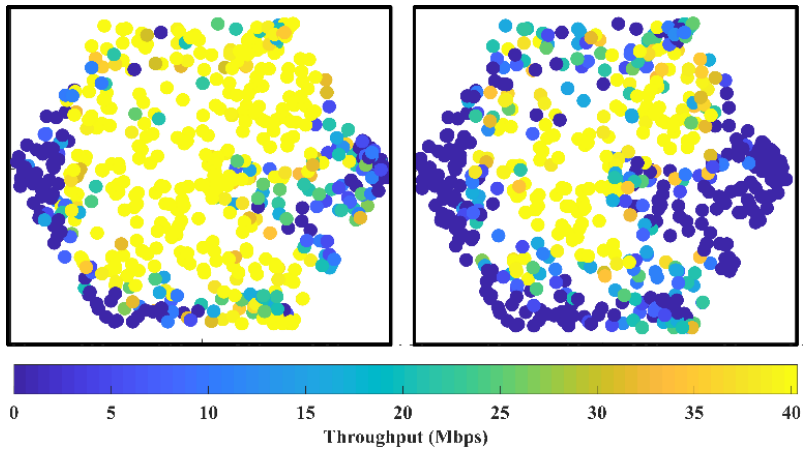

a) $\mathrm{SISO}-800 \mathrm{MHz}$

b) $\mathrm{SISO}-2600 \mathrm{MHz}$
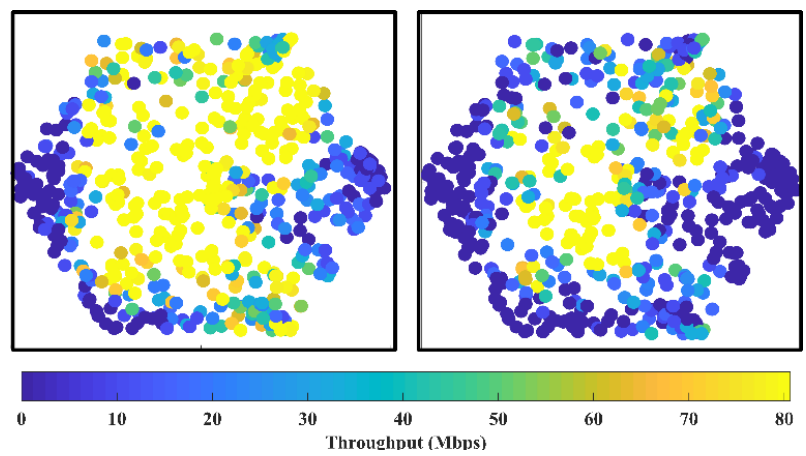

c) $2 \times 2 \mathrm{MIMO}-800 \mathrm{MHz}$

d) $2 \times 2$ MIMO-2600 MHz
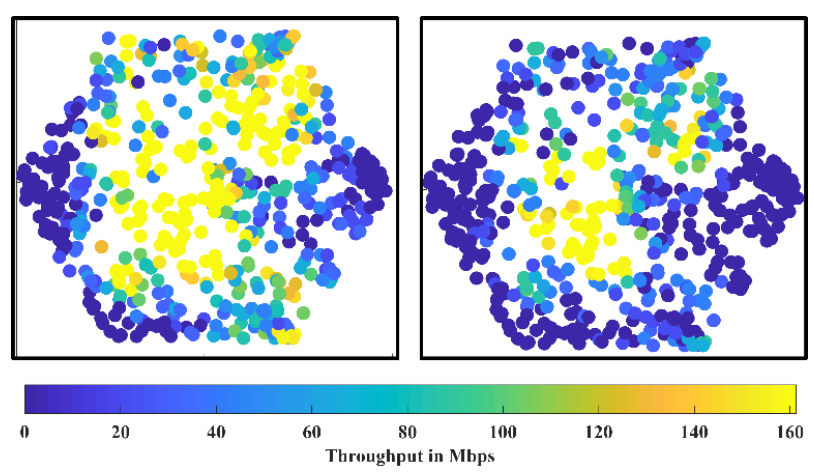

e) $4 \times 4 \mathrm{MIMO}-800 \mathrm{MHz}$

f) $4 \times 4$ MIMO-2600 MHz

Figure 10: UEs throughput coverage map for one cell for component carriers of $800 \mathrm{MHz}$ and $2600 \mathrm{MHz}$

\section{CONCLUSIONS}

This paper has presented propagation channel statistics for a large number of UEs in two different frequency bands and three different antenna systems in urban environment using the enhanced 3D ITU-R channel model. An RBIR abstraction model for the LTEAdvanced downlink physical layer was used to determine the throughput and PER versus SNR at each UE. Simulation results shows that the efficiency of carrier aggregation efficiency is engaged with increasing the number of antennas. The efficiency of carrier aggregation decreases as the number of spatial stream increase due to increased inter stream interference. Accordingly, the following are recommended to increase the efficiency of 
the carrier aggregation without increasing the BS total transmit power:

- Heterogenous deployment with larger cell size for the $800 \mathrm{MHz}$ band and small cells for the $2600 \mathrm{MHz}$ band

- Decrease the cell radius of the $800 \mathrm{MHz}$ band to $500 \mathrm{~m}$ instead of $1000 \mathrm{~m}$.

- Choose a cell radius of $200 \mathrm{~m}$ for the $2600 \mathrm{MHz}$ band.

This study can be extended in the future to consider the aforementioned recommendation and other parameters such as interference and different spacing between antenna elements.

\section{REFERENCE}

[1] E. Dahlman, S. Parkvall, and J. Skold, 4G LTE/LTE-Advanced for Mobile Broadband, Elsevier/Academic Press, Oxford, 2011.

[2] 3GPP TR 36.850: "Evolved Universal Terrestrial Radio Access (E-UTRA): Inter-band Carrier Aggregation," V11.0.0, 2013.

[3] S. Lee, S. Hyeon, J. Kim, H. Roh and W. Lee, "The Useful Impact of Carrier Aggregation: A Measurement Study in South Korea for Commercial LTE-Advanced Networks," IEEE Vehicular Technology Magazine, 12 (1), pp. 55-62, 2017.

[4] 3GPP TSG-RAN-1 Meeting \#35, R1-03-1298, "Effective SIR Computation for OFDM SystemLevel Simulations," 2003.

[5] L. Wan, S. Tsai, and M. Almgren, "A FadingInsensitive Performance Metric for a Unified Link Quality Model," In Proceedings of the IEEE Wireless Communications and Networking Conference (WCNC), pp. 2110 - 2114, 2006.

[6] A. Ameen, E. Mellios, A. Doufexi, N. Dahnoun, and A. Nix, "LTE Advanced Downlink Throughput Evaluation in the $3 \mathrm{G}$ and TV White Space Bands," In Proceedings of the IEEE International Symposium on Personal Indoor and Mobile Radio Communications (PIMRC), pp. 771-775, 2013.

[7] T. Huang, J. Yuan, X. Cheng and W. Lei, "Advanced Link-to-System Modeling of MMSESIC Receiver in MIMO-OFDM Systems," In Proceedings of the IEEE International Conference on Signal Processing and Communication Systems (ICSPCS), pp. 1-6, 2015.

[8] A. Medles and C. Valadon, "Iterative MIMO Effective SNR Mapping for ML Decoder," In Proceedings of the IEEE Vehicular Technology Conference (VTC), pp. 1-5, 2015.

[9] R. Almesaeed, A. Ameen, A. Doufexi, N. Dahnoun, and A. Nix, "A Comparison Ctudy of 2D and 3D ITU Channel Model," In Proceedings of the IEEE Wireless Days (WD), pp. 1-7, 2013.

[10] R. Almesaeed, A. Ameen, E. Mellios, A. Doufexi, and A. Nix, "3D Channel Models: Principles, Characteristics, and System Implications," IEEE Communications Magazine, 55 (4), pp. 152-159, 2017.
[11] ITU-R M.2135-1, "Guidelines for Evaluation of Radio Interface Technologies for IMT-Advanced”, 2009.

[12] E. Mellios, Z. Mansor, G. Hilton, A. Nix, and J. McGeehan, "Impact of Antenna Pattern and Handset Rotation on Macro-Cell and Pico-Cell Propagation in Heterogeneous LTE networks," In Proceedings of the IEEE International Symposium on Antennas and Propagation, pp. 1-2, 2012.

[13] R. Almesaeed and A. Ameen, "Enhanced 3D ITU Channel Model", sourceforge.net, source code, Jan., 2, 2016. [Online]. Available: http://enhanced-3d-ituchannel-model.sourceforge.net [Accesed: April. 12, 2017].

[14] 3GPP TS 36.942, "Evolved Universal Terrestrial Radio Access (E-UTRA): Radio Frequency (RF) System Scenarios," V10.2.0, 2010.

[15] A. Doufexi, E. Tameh, A. Nix, S. Armour, and A. Molina, "Hotspot Wireless LANs to Enhance the Performance of $3 \mathrm{G}$ and Beyond Cellular Networks," IEEE Communications Magazine, 41 (7), pp.58-65, 2003.

[16] 3GPP TS 36.101: "Evolved Universal Terrestrial Radio Access (E-UTRA): User Equipment (UE) Radio Transmission and Reception," V10.10.0, 2013. 